\title{
Optimal Energy Consumption for Mobile Manipulators Executing Door-Opening Task
}

\author{
Changyou Ma $\mathbb{D},{ }^{1,2}$ Haibo Gao $\left(\mathbb{D},{ }^{1}\right.$ Liang Ding $\left(\mathbb{D},{ }^{1}\right.$ Jianguo Tao, ${ }^{1}$ Kerui Xia, ${ }^{1}$ \\ Haitao Yu, ${ }^{1}$ and Zongquan Deng ${ }^{1}$ \\ ${ }^{1}$ State Key Laboratory of Robotics and System, Harbin Institute of Technology, Harbin 150001, China \\ ${ }^{2}$ College of Mechanical Engineering, Jiamusi University, Jiamusi 154007, China \\ Correspondence should be addressed to Haibo Gao; gaohaibo@hit.edu.cn and Liang Ding; liangding@hit.edu.cn
}

Received 17 November 2017; Accepted 26 March 2018; Published 25 June 2018

Academic Editor: Marco Spadini

Copyright (C) 2018 Changyou Ma et al. This is an open access article distributed under the Creative Commons Attribution License, which permits unrestricted use, distribution, and reproduction in any medium, provided the original work is properly cited.

As a substitute for humans, the mobile manipulator has become increasingly vital for on-site rescues at Nuclear Power Plants (NPPs) in recent years. The high energy efficiency of the mobile manipulator when executing specific rescue tasks is of great importance for the mobile manipulator. This paper focuses on the energy consumption of a robot executing the door-opening task, in a scenario mimicking an NPP rescue. We present an energy consumption optimization scheme to determine the optimal base position and joint motion of the manipulator. We developed a two-step procedure to solve the optimization problem, taking the quadric terms of the joint torques as the objective function. Firstly, the rotational motion of the door is parameterized by using piecewise fifth-order polynomials, and the parameters of the polynomials are optimized by minimizing the joint torques at the specified base position using the Quasi-Newton method. Second, the global optimal movement of the manipulator for executing the door-opening task is acquired by means of searching a grid for feasible base positions. Comprehensive door-opening experiments using a mobile manipulator platform were conducted. The effectiveness of the proposed method has been demonstrated by the results of physical experiments.

\section{Introduction}

Mobile manipulators, as a replacement for humans, play a key role when they perform rescue tasks in the extreme environment of a nuclear power plant (NPP), such as door-opening and turning a valve. The energy optimization of rescue tasks performed by a robotic arm, in particular that of the fundamental task of door-opening, is of great importance when the energy supply is restricted by the capacity of the battery with which the robot can be equipped, unlike in traditional methods where energy is supplied via a cable.

The Fukushima Daiichi NPP accident in Japan on March 11, 2011, was triggered by an earthquake of magnitude 9.0 and the resultant tsunami marked the beginning of the worst nuclear accident of the last two decades [1-3]. Nuclear power is an important resource and (NPPs) have recently undergone rapid development in China [4]. The complexity of the NPP's structure, the radioactive working environment, and some special characteristics, such as high temperatures and high pressure, render nuclear disaster rescue very difficult. The dangers caused by such an accident mean that mobile robot plays an important role in the NPP rescue process. After the Fukushima Daiichi NPP accident, the Defense Advanced Research Projects Agency (DARPA) initiated a new challenge in 2012, the DARPA Robotics Challenge (DRC) [5], including eight rescue tasks to test the capabilities of teams, as well as of individuals. The tasks were ranked by DARPA in terms of difficulty as Valve (easiest), Terrain and Hose (easier), Door, Debris, Wall, and Ladder (harder), and Vehicle (hardest) [6].

In this paper, we focus on the energy consumption of a mobile manipulator performing the door-opening task. Nagatani and Yuto proposed a method that allows a mobile manipulator, named the "YAMABICO-10" robot, to open a door and pass through the doorway. In their approach, they applied to the mobile manipulator control system the concept of action primitives, which control the "YAMABICO-10" 
robot according to sequences of planned motion primitives. However, each action primitive was designed with an error adjustment mechanism to handle the accumulated positioning error of the mobile base [7-9]. Peterson et al. presented the design and implementation of a door-opening controller using a hybrid dynamic system model; when using this simple controller, the radius and center of rotation of the door are estimated online. The results of their experiments demonstrated that off-the-shelf algorithms for force/torque control are very effective for solving the task of grasping the handle and opening the door [10]. Chung et al. [11] proposed a control strategy for the door-opening procedure executed by a service robot, PSR1, which utilized a three-fingered robot hand for grasping the door handle. Two active-sensing strategies were proposed to estimate the kinematic parameters in a real environment. An integrated strategy of motion coordination was presented based on the components of three subsystems: a robotic hand, a robotic arm, and a mobile robot. The force and position control were successfully achieved by using the contact force of the three-fingered robotic hand during the door-opening procedures.

Ahmad et al. designed a modular and reconfigurable robot (MRR) mounted on a wheeled mobile platform [12, 13]. They proposed a new method that utilizes the multiple working modes of the MRR modules to prevent the occurrence of large internal forces that arise because of positioning errors or imprecise modeling of the robot or its environments. By selectively switching the joints of the MRR to work in passive mode during the door-opening operation, the controller design was significantly simplified. Zhang et al. [14] presented a multiple mode control system of a two-degree-of-freedom (DOFs) compact wrist that can work in active mode with position or torque control, or in passive mode with wristenvironment interactive force compensation. They verified in their door-opening experiments that the wrist could move freely without generating excessive internal force. Kobayashi et al. [15] designed a rescue robot series named UMRS. The robot had a special end-effector, which can grip and rotate cylindrical type and lever type door handles. The robot equipped with a door-opening system was capable of moving freely through rooms, even if there were doors between them. Klingbeil et al. [16] proposed a method that used vision to identify a small number of key positions, such as the axis of rotation of the door handle, and the end-point of the door handle, to allow a manipulator to open various types of doors without prior knowledge of their parameters. Karayiannidis et al. [17] developed an algorithm that can be implemented in a velocity-controlled manipulator, the end- effector which is equipped with force sensing capabilities. The method consists of a velocity controller, which uses force measurements and an estimation of the radial direction based on adaptive estimates of the position of the door hinge. The control action can be decomposed into an estimated radial and tangential direction following the concept of hybrid force/motion control. Endres et al. [18] presented an approach for learning a dynamic model of a door from sensor observations and utilizing it for effectively swinging the door open to the desired angle. The learned models enable the realization of dynamic door-opening strategies and reduce the complexity of the door-opening task.
As proposed in [19], a power efficiency estimation-based health monitoring and fault detection method has been developed for a modular and reconfigurable robot (MRR). The power efficiency of each of the robot's joints is measured using sensors. Luo et al. [20] presented the Lagrange interpolation method to express each joint trajectory function to realize trajectory planning that achieves energy minimization of industrial robotic manipulators. Field and Stepanenko presented an iterative dynamic programming method that is modified to perform a series of dynamic programming, passing over a small reconfigurable grid that covers only a portion of the solution space at any one pass, to plan minimum energy consumption trajectories for robotic manipulators [21]. Liu et al. [22] proposed the fourth-order Runge-Kutta method, multiple shooting methods, and traversing method to solve optimal energy trajectory planning for palletizing robot.

The objective of this study was to develop an energy consumption optimization method for the door-opening procedure. The main contributions of this paper are summarized as follows. An energy consumption optimization scheme that finds the optimal base position and joint motion of the manipulator for optimizing energy consumption is presented. Since the end-effector trajectory is assigned according to the trajectory of the door handle, the problem of searching the optimal manipulator movement by using the parameters of the door's fifth-order splines transforms to a simple parametric optimization problem at each base position. The global optimal energy-efficient movement of the robotic arm is finally obtained by exhaustively searching the entire base position grid.

The rest of the paper is organized as follows. In Section 2, we introduce the door-opening task and establish the energy consumption optimization objective function. Section 3 provides a description of the mobile modular robot and introduces the kinematic and the dynamics model of the manipulators. In the Section 4, we address the optimization method and describe numerical simulations. The results of our experiments are discussed in Section 5, and conclusions are presented in Section 6.

\section{Formulation of Optimization Problem}

2.1. Door-Opening Operation. In this section, we propose a door- opening method. The following assumptions were made: (1) the door-opening and door handle rotating directions are known (the door is opened toward the left-side and the door handle is rotated toward the right-side); (2) the door axis of rotation is perpendicular to the floor; (3) the door moves in the horizontal $x y$ plane; $(4)$ the mobile platform travels on the ground, which can always be adjusted for a structured laboratory environment; and (5) the axis of rotation of the first manipulator module is perpendicular to the ground.

A brief explanation of the door-opening procedure is as follows.

(a) The mobile manipulator moves such that it is positioned in front of the door and grasps the door handle; 


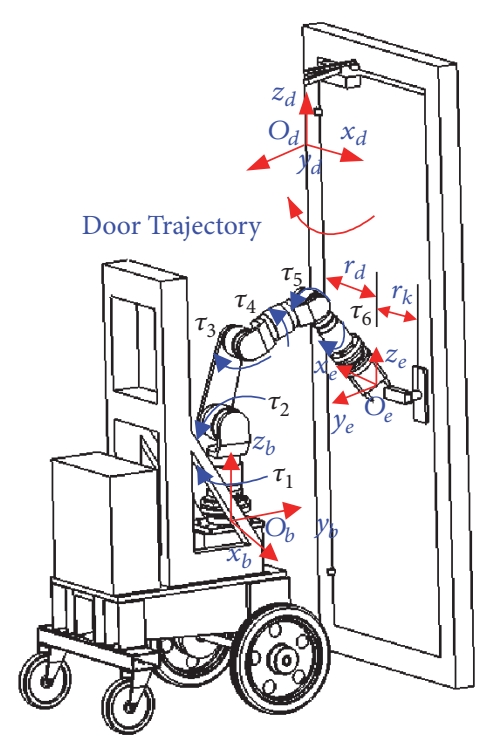

Figure 1: Model of the door-opening procedure with a mobile manipulator.

(b) The mobile base remains static. The manipulator realizes turning the door handle by tracking a planned trajectory;

(c) The mobile base still remains static. The manipulator realizes pulling the door by tracking a planned trajectory in the horizontal $x y$ plane.

A model of the door-opening procedure using the mobile manipulator is depicted in Figure 1. The figure shows the origin of the reference frame, $\left\{O_{d}\right\}$, set at the intersection point of the door hinge, the horizontal plane that crosses the origin of the reference frame of the mobile manipulator, $\left\{\mathrm{O}_{b}\right\}$, and the reference frame, $\left\{O_{e}\right\}$, located at the position of the manipulator end-effector, which is used to grasp the door handle.

In order to plan the path of the mobile manipulator, we first need to know the accurate value of the door handle radius $\left(r_{k}\right)$ and the door radius $\left(r_{d}\right)$, the initial base position of the mobile manipulator $\left(x_{b}, y_{b}, z_{b}\right)$, and the position of the endeffector, which holds the door handle firmly $\left(x_{e}, y_{e}, z_{e}\right)$. The door motion is conformed to follow the door trajectory in the $x y$ plane with the center of rotation at $\left(x_{d}, y_{d}\right)$ and a radius $r_{d}$ as shown in the Figure 1. The trajectory radius of pulling the door is derived as follows:

$$
\left[x_{e}(t)-x_{d}\right]^{2}+\left[y_{e}(t)-y_{d}\right]^{2}=r_{d}^{2} \text {. }
$$

2.2. Path Planning of Door-Opening. In this section, we focus on pulling the door handle to open the door. During this procedure, the home position of the mobile base, the door radius, and the height of the door handle are measured. These measured parameters are then used for planning the path of the mobile manipulator that allows it to open the door to the desired angle.

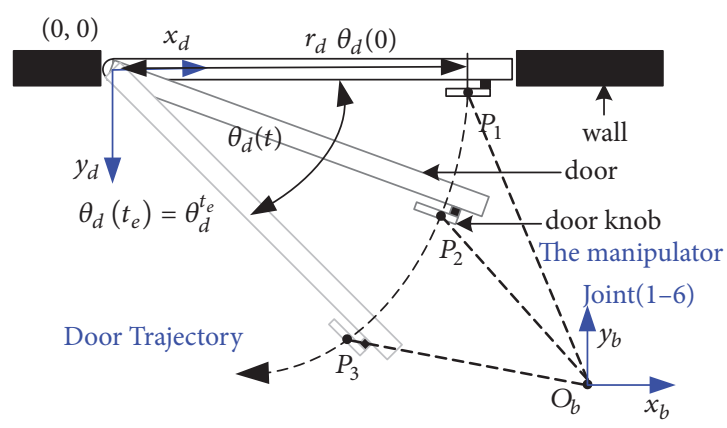

Figure 2: Planar model of pulling door.

The mobile manipulator achieves the action of pulling the door by tracking the circle arc represented by the door trajectory, as shown in Figure 2. The arc of the door trajectory can be acquired by using the interpolation points $P_{1}, P_{2}$, and $P_{3}$. In Figure 2, we show the optimal base position $\left(x_{b}, y_{b}\right)$ during the door-opening procedure. The mobile manipulator approaches the door and stops at any position near the door. The end-effector is fixed at the same position as the door handle during the door-opening procedure, and each joint of the manipulator rotates in accordance with the motion planning. The door is pulled in the horizontal $x y$ plane, and the initial and final angles of the door are consistent. The angles of Joints $1,2,3,4,5$, and 6 are denoted as $\theta_{1}, \theta_{2}, \theta_{3}, \theta_{4}$, $\theta_{5}$, and $\theta_{6}$, respectively. The input torques of each joint of the manipulator are expressed as $\tau_{1}, \tau_{2}, \tau_{3}, \tau_{4}, \tau_{5}$, and $\tau_{6}$, respectively. The angles and the torques are represented in matrix forms as $\boldsymbol{\theta}=\left[\theta_{1}, \theta_{2}, \theta_{3}, \theta_{4}, \theta_{5}, \theta_{6}\right]^{\mathrm{T}}$ and $\boldsymbol{\tau}=\left[\tau_{1}, \tau_{2}, \tau_{3}, \tau_{4}, \tau_{5}\right.$, $\left.\tau_{6}\right]^{\mathrm{T}}$. The angle of the door is expressed as $\theta_{d}$, and the conditions on $\theta_{d}$ at the start time, $t=0$, and the end time, $t=t_{e}$, are written as

$$
\begin{aligned}
& \left.\left(\theta_{d}, \dot{\theta}_{d}\right)\right|_{t=0}=(0,0), \\
& \left.\left(\theta_{d}, \dot{\theta}_{d}\right)\right|_{t=t_{e}}=\left(\theta_{d}^{t_{e}}, 0\right) .
\end{aligned}
$$

The positions of the end-effector at points $\mathbf{P}_{1}, \mathbf{P}_{2}$ and $\mathbf{P}_{3}$ are computed as follows:

$$
\begin{gathered}
\theta_{d}=\frac{\theta_{\text {door }}}{3} n, \quad n=1,2,3 \\
\mathbf{P}_{1,2,3}=\left[\begin{array}{c}
p_{x} \\
p_{y} \\
p_{z}
\end{array}\right]=\left[\begin{array}{c}
r_{d}\left(1-\cos \theta_{d}\right) \\
y_{1}-r_{d} \sin \theta_{d} \\
z_{1}
\end{array}\right],
\end{gathered}
$$

where $r_{d}$ denotes the rotation radius of the door, $\theta_{d}$ denotes the rotation angle of the door when reaching points $\mathbf{P}_{1}, \mathbf{P}_{2}$, and $\mathbf{P}_{3}$, and $\theta_{\text {door }}$ denotes the rotation angle of the door that allows the mobile manipulator to enter the doorway.

The orientations of the end-effector and the door are the same during the process of pulling the door. When the position of the end-effector remains constant with respect to the door handle, as shown in Figure 1, the variation in the rotation matrix during the process denoted by ${ }_{e}^{0} \mathbf{R}_{d}^{(0)} \cdot{ }_{e}^{0} \mathbf{R}_{d}^{(0)}$ 


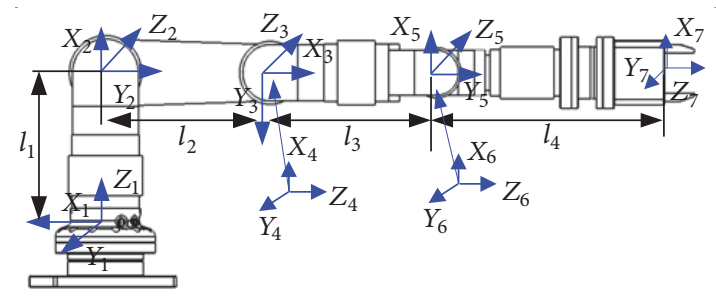

FIgURE 3: Kinematics configuration of the 6 DoF manipulator.

denotes the initial rotation matrix of the end-effector after turning the door handle, which can be acquired by using forward kinematics [23]. Then, at time $t$, this matrix is multiplied by a rotation matrix parameterized by the rotation angle of the door $\theta_{d}^{(t)}$ :

$$
{ }_{e}^{0} \mathbf{R}_{d}^{(t)}=\left[\begin{array}{ccc}
\cos \theta_{d}^{(t)} & -\sin \theta_{d}^{(t)} & 0 \\
\sin \theta_{d}^{(t)} & \cos \theta_{d}^{(t)} & 0 \\
0 & 0 & 1
\end{array}\right]{ }_{e}^{0} \mathbf{R}_{d}^{(0)}
$$

2.3. Objective Formulation of Energy Consumption. The energetic cost is an important metric of the energy consumption of the manipulator. We experimentally evaluated the effect of different base positions of the robot on the energy consumption of the manipulator during the door-opening procedure. The input energy $E_{m}$ of the motor is as follows:

$$
E_{m}=\int_{0}^{t_{e}} U_{\text {in }} \cdot I_{m} d t,
$$

where $U_{\text {in }}$ is the power supply voltage and $I_{m}$ is the instantaneous current of the DC motor. The total energy consumed by the robot's actuators includes the generated mechanical power $\left(P_{\text {mech }}\right)$, heat power $\left(P_{\text {heat }}\right)$, and power losses $\left(\Delta_{\text {loss }}\right)$. This can be described as

$$
P_{\text {in }}=P_{\text {mech }}+P_{\text {heat }}+\Delta_{\text {loss }} \text {. }
$$

The mechanical power generated by each actuator is related to the rotation angular velocity and torque. Therefore, the total instantaneous mechanical power during the movement of the manipulator can be stated as

$$
P_{\text {mech }}=\sum_{j=1}^{6} \tau_{j} \cdot \omega_{j} \text {, }
$$

where $P_{\text {mech }}$ is the total mechanical power of the actuators, $\tau_{j}$ is the torque of each actuators in $\mathrm{N} \cdot \mathrm{m}, \omega_{j}$ is the rotation angular velocity of motors in $\mathrm{rad} / \mathrm{s}$, and $j$ is the number of manipulator joints.

The torque $\tau$ of each actuator is related to the torque constant $K_{m}$, where, $i$ is the gear ratio of the joint, $\eta$ is the efficiency of the transmission mechanism, and $I_{m}$ is the instantaneous current; $\tau$ is provided by

$$
\tau=K_{m} \cdot I_{m} \cdot i \cdot \eta
$$

As shown in (7), the mechanical power consists of the torque of each actuators and the rotation angular velocity of the motors. However, the angular velocity range of the joint trajectory planning of the manipulator is relatively small. Therefore, the problem of energy optimization becomes the joint torque optimization problem. For the trajectory optimization procedure, the integral of squared joint torques as the cost function is known from studies in the literature [25]. We introduce the following objective function as a standard for optimization.

$$
E_{\text {con }}(\varsigma)=\sum_{j=1}^{6} \int_{t=0}^{t_{\mathrm{e}}} \tau_{j}^{2} d t .
$$

Here, $\varsigma$ denotes the optimal parameter, which is chosen as the position of mobile platform base and the trajectory of the door angle. To solve the problem of energy consumption optimization during the door-opening procedure, we determine the parameters that minimize $E_{\text {con }}$ :

$$
\varsigma^{*}=\arg \min E_{\mathrm{con}}
$$

\section{Description of the Mobile Manipulator}

3.1. Kinematics of the Robot. The kinematics model configuration of the 6-DOF Schunk modular manipulator is shown in Figure 3.

The forward kinematics is developed by using the D-H method and represented by ${ }_{0}^{6} \mathrm{~T}$.

$$
{ }_{0}^{6} \mathbf{T}=\left[\begin{array}{cccc}
\mathbf{R}_{3 \times 3} & & \mathbf{p}_{1 \times 3} \\
0 & 0 & 0 & 1
\end{array}\right]=\left[\begin{array}{cccc}
r_{11} & r_{12} & r_{13} & p_{x} \\
r_{21} & r_{22} & r_{23} & p_{y} \\
r_{31} & r_{32} & r_{33} & p_{z} \\
0 & 0 & 0 & 1
\end{array}\right],
$$

where $\mathbf{R}_{3 \times 3}$ and $\mathbf{p}_{1 \times 3}$ are the rotation and translation matrix. Based on (11), the joint angles by inverse kinematics calculations can be derived as 


$$
\left[\begin{array}{c}
\theta_{1} \\
\theta_{2} \\
\theta_{3} \\
\theta_{4} \\
\theta_{5} \\
\theta_{6}
\end{array}\right]=\left[\begin{array}{c}
\operatorname{atan} 2\left(p_{y}, p_{x}\right)-\operatorname{atan} 2\left(0, \pm \sqrt{p_{x}^{2}+p_{y}^{2}}\right) \\
\theta_{23}-\theta_{3} \\
\operatorname{atan} 2\left(K, \pm \sqrt{l_{3}^{2}-K^{2}}\right) \\
\operatorname{atan} 2\left[-r_{13}\left(c_{1} c_{23} c_{4}+s_{1} s_{4}\right)-r_{23}\left(s_{1} c_{23} c_{4}-c_{1} s_{4}\right) r_{33} s_{23} c_{4},-r_{13} c_{1} s_{23}-r_{23} s_{1} s_{23}-r_{33} c_{23}\right] \\
\operatorname{atan} 2\left(s_{6}, c_{6}\right)
\end{array}\right]
$$

where

$$
\begin{aligned}
K & =\frac{p_{x}^{2}+p_{y}^{2}+p_{z}^{2}-a_{2}^{2}-l_{3}^{2}}{2 a_{2}}, \\
\theta_{23} & =\operatorname{atan} 2\left[-a_{2} c_{3} p_{z}\right. \\
& -\left(c_{1} p_{x}+s_{1} p_{y}\right)\left(l_{3}-a_{2} s_{3}\right),\left(a_{2} s_{3}-l_{3}\right) p_{z} \\
& \left.-\left(a_{3}+a_{2} c_{3}\right)\left(c_{1} p_{x}+s_{1} p_{y}\right)\right], \\
& c_{i}=\cos \left(\theta_{i}\right), s_{i}=\sin \left(\theta_{i}\right) .
\end{aligned}
$$

Because of the redundancy, there are eight groups of joint angles through the inverse kinematics for giving the position of the end-effector. An algorithm that can minimize the Euler distance in joint space from the initial state should be chosen from the eight solutions [23]. The Jacobin matrix of the 6DOF manipulator is

$$
\mathbf{J}=\left[\begin{array}{cccccc}
J_{11} & J_{12} & J_{13} & J_{14} & J_{15} & J_{16} \\
J_{21} & J_{22} & J_{23} & J_{24} & J_{25} & J_{26} \\
J_{31} & J_{32} & J_{33} & J_{34} & J_{35} & J_{36} \\
0 & 0 & 0 & J_{44} & J_{45} & J_{46} \\
0 & 0 & 0 & J_{54} & J_{55} & 0 \\
0 & 0 & 0 & 0 & 0 & 1
\end{array}\right]
$$

Based on (14), the angular velocity $\dot{\theta}_{i}$ and acceleration $\ddot{\theta}_{i}$ of the joint can be derived as

$$
\begin{aligned}
& {\left[\begin{array}{llllll}
\dot{\theta}_{1} & \dot{\theta}_{2} & \dot{\theta}_{3} & \dot{\theta}_{4} & \dot{\theta}_{5} & \dot{\theta}_{6}
\end{array}\right]^{\mathrm{T}}=\mathbf{J}^{-1} \dot{\mathbf{P}}} \\
& {\left[\begin{array}{llllll}
\ddot{\theta}_{1} & \ddot{\theta}_{2} & \ddot{\theta}_{3} & \ddot{\theta}_{4} & \ddot{\theta}_{5} & \ddot{\theta}_{6}
\end{array}\right]^{\mathrm{T}}=\mathbf{J}^{-1} \ddot{\mathbf{P}}+\mathbf{J}^{-1} \mathbf{\mathbf { P }},}
\end{aligned}
$$

where $\mathbf{P}$ is the position and orientation vector of the endeffector of the manipulator.

3.2. Dynamics of the Robot. From the inverse kinematics in the previous subsection, the equations for the motion of the system can be written by using $\theta_{d}(t)$ :

$$
\left[\tau_{1}, \tau_{2}, \tau_{3}, \tau_{4}, \tau_{5}, \tau_{6}, 0\right]^{\mathrm{T}}-\mathbf{B F}=\boldsymbol{\Psi}\left(\theta_{d}, \dot{\theta}_{d}, \ddot{\theta}_{d}\right),
$$

where $\mathbf{F}=\left[F_{1}, F_{2}, F_{3}, M_{1}, M_{2}, M_{3}\right]^{\mathrm{T}}$ is the force and torque of the $x y z$ direction used for grasping the door handle. $\Psi$ denotes the dynamics parameter matrix of the door in (16), including the symmetric positive definite manipulator inertia matrix $\mathbf{M}\left(\theta_{d}\right)$, the vector of centripetal and Coriolis torques $\mathbf{C}\left(\theta_{d}, \dot{\theta}_{d}\right)$, and the vector of gravitational torques $\mathbf{g}\left(\theta_{d}\right)$. The matrix $\mathbf{B}$ can be obtained as

$$
[\mathbf{B}]^{\mathrm{T}}=\left[\frac{\partial \Phi}{\partial \theta}, \frac{\partial \Phi}{\partial \theta_{d}}\right]=\left[\mathbf{J}, \frac{\partial \Phi}{\partial \theta_{d}}\right]
$$

where $\Phi$ is a function of the relationship between the angle of the door-opening and the joint angle of the manipulator. $\Phi\left(\theta, \theta_{d}\right)=f_{2}(\theta)-f_{1}\left(\theta_{d}\right)=0$.

$$
\begin{aligned}
f_{1}\left(\theta_{d}\right) & =\left[\begin{array}{c}
r_{d} \cos \left(\theta_{d}\right) \\
r_{d} \sin \left(\theta_{d}\right) \\
z_{d}
\end{array}\right], \\
f_{2}(\theta) & =\left[\begin{array}{c}
x_{b}+d_{4}\left(c_{2} s_{1} s_{3}+c_{3} s_{1} s_{2}\right)+d_{6}\left(s_{5}\left(c_{1} s_{4}-c_{4}\left(s_{1} s_{2} s_{3}-c_{2} c_{3} s_{1}\right)\right)+c_{5}\left(c_{2} s_{1} s_{3}+c_{3} s_{1} s_{2}\right)\right)+a_{2} s_{1} s_{2} \\
y_{b}+d_{6}\left(s_{5}\left(s_{1} s_{4}+c_{4}\left(c_{1} s_{2} s_{3}-c_{1} c_{2} c_{3}\right)\right)-c_{5}\left(c_{1} c_{2} s_{3}+c_{1} c_{3} s_{2}\right)\right)-d_{4}\left(c_{1} c_{2} s_{3}+c_{1} c_{3} s_{2}\right)-a_{2} c_{1} s_{2} \\
z_{d}
\end{array}\right],
\end{aligned}
$$

where $z_{d}$ is the coordinate value of the $z$ direction in the coordinate system of the door, $c_{i}$ and $s_{i}$ are the abbreviations for $\cos \left(\theta_{i}\right)$ and $\sin \left(\theta_{i}\right)$, respectively, and $l_{1}, l_{2}, l_{3}$, and $l_{4}$ are the lengths of the links in Figure 3. 
We cannot determine $\tau$ in (16), which means that the system is indeterminate. F can be measured by using the six-axis $\mathrm{F} / \mathrm{T}$ sensor, and the forward six rows of (16) can be rewritten as

$$
\boldsymbol{\tau}-\mathbf{J}^{\mathrm{T}} \mathbf{F}=\mathbf{M}(\theta) \ddot{\theta}+\mathbf{V}(\theta, \dot{\theta})+\mathbf{G}(\theta),
$$

where $\mathbf{M}(\theta)$ is the $6 \times 6$ inertia matrix of the manipulator, $\mathbf{V}(\theta, \dot{\theta})$ is the $6 \times 1$ vector of the centrifugal force and Coriolis force, and $\mathbf{G}(\theta)$ is the $6 \times 1$ vector of the gravity.

\section{Optimization Method}

In this part, we focus on the optimal method for the dooropening procedure. Since $\theta_{d}(t)$ is an infinite dimensional parameter, it is difficult to find the optimal solution that minimizes $E_{\text {con }}$ rigorously. Therefore, we approximate $\theta_{d}(t)$ as the fifth-order spline functions of time and find the coefficients of splines that minimize the cost function. The position of the mobile manipulators base $\left(x_{b}, y_{b}\right)$ is discretized into a grid, and at each grid point, the quasi-optimal motion of the door is calculated by using the spline functions.

4.1. Optimal Motion of Door. We divide the time interval $\left[0, t_{e}\right]$ by $n$ and assume that the trajectory of $\theta_{d}(t)$ in each time interval $\left[t_{i}, t_{i+1}\right]\left(i=0, \ldots, n-1\right.$ and $t_{j}=j t_{e} / n$ for $j=0, \ldots, n)$ is expressed by a fifth-order polynomial function of time, $\mathbf{X}_{i}(t)$, as

$$
\begin{aligned}
\mathbf{X}_{i}(t)= & \theta_{d i}+a_{1 i}\left(t-t_{i}\right)+a_{2 i}\left(t-t_{i}\right)^{2}+a_{3 i}\left(t-t_{i}\right)^{3} \\
& +a_{4 i}\left(t-t_{i}\right)^{4}+a_{5 i}\left(t-t_{i}\right)^{5}
\end{aligned}
$$

where $a_{1 i}, a_{2 i}, a_{3 i}, a_{4 i}$, and $a_{5 i}$ are the coefficients of the polynomial. In order to make the input torque $\tau$ continuous, we choose the function such that $\mathbf{X}_{i}\left(t_{i+1}\right)=\mathbf{X}_{i+1}\left(t_{i+1}\right), \dot{\mathbf{X}}_{i}\left(t_{i+1}\right)=$ $\dot{\mathbf{X}}_{i+1}\left(t_{i+1}\right), \ddot{\mathbf{X}}_{i}\left(t_{i+1}\right)=\ddot{\mathbf{X}}_{i+1}\left(t_{i+1}\right)$, for $i=0, \ldots, n-2$, and (20) satisfies (2). The coefficients $a_{1 i}, a_{2 i}$, and $a_{3 i}$ are constant. Therefore, there are $3 n-1$ independent parameters, and they can be shown as

$$
\mathbf{Z}=\left(\theta_{d 1}, \ldots, \theta_{d n-1}, a_{40}, \ldots, a_{4 n-1}, a_{50}, \ldots, a_{5 n-1}\right) .
$$

We assume that the door angle $\theta_{d}(t)$ is a monotonically increasing function at $t=t_{i}$ with constraint $0 \leq \theta_{d 0} \leq \cdots \leq$ $\theta_{d n-1} \leq \theta_{d t_{e}}$. At each position of the mobile manipulator's base, we search for the values of $\mathbf{Z}$ that minimize the $E_{\text {con }}$ by using the Quasi-Newton method. We can find a vector $\mathbf{h}=$ $\left[h_{1}, h_{2}, h_{3}, h_{4}, h_{5}, h_{6}, h_{7}\right]^{\mathrm{T}}$ that conforms to $\mathbf{h}^{\mathrm{T}} \mathbf{B}=0$. Eq. (16) is multiplied by the vector $h$, and thus, we obtain

$$
h_{1} \tau_{1}+h_{2} \tau_{2}+h_{3} \tau_{3}+h_{4} \tau_{4}+h_{5} \tau_{5}+h_{6} \tau_{6}=\mathbf{h}^{\mathrm{T}} \boldsymbol{\Psi} .
$$

The manipulator joint torque $\tau$ satisfying (22) can obtain a specified motion of the door $\theta_{d}(t)$. When $\|\tau\|$ is minimized, $\tau$ can be shown as

$$
\boldsymbol{\tau}=k_{\tau}\left[h_{1}, h_{2}, h_{3}, h_{4}, h_{5}, h_{6}\right]^{\mathrm{T}},
$$

where $k_{\tau}$ is a scalar parameter. By using (23), we can calculate $\tau$ uniquely. We propose a method, as shown in Algorithm 1, to acquire the optimal motion of the door and the corresponding torque of each joint.
4.2. Optimal Position of the Mobile Manipulator's Base. We determine the optimal position of the mobile manipulator's base and the end-effector's grasp by using the exhaustive method. The region of $\left(x_{b}, y_{b}\right)$, defined by $\left[x_{b}^{\min }, x_{b}^{\max }\right] \times$ $\left[y_{b}^{\min }, y_{b}^{\max }\right]$, is divided into a grid, where each rectangle is given by $\Delta x \times \Delta y$. By calculating the objective function at each grid point using the method in Section 4.1, with a set of $\left[\tau_{i}, X_{b i}, Y_{b i}\right]$, we can determine the optimal position of the mobile manipulator's base. The scheme for optimizing the position is shown in Algorithm 2.

4.3. Numerical Simulations. In this section, we describe the acquisition of the optimal solution by using numerical simulations. We used MATLAB to find the optimal values of $Z$ under the constraint that $0 \leq \theta_{d 0} \leq \cdots \leq \theta_{d n-1} \leq$ $\theta_{d t}$. The open angle of the door, $\theta_{d}(t)$, was set to be $\pi / 3$ at $t_{e}=20 \mathrm{~s}$. The time of the door pulling interval $[0,20]$ was divided into ten subintervals; that is, $n=10$. The measured length of the door was $0.85[\mathrm{~m}]$. The mass and inertia of the door were set to $35.7[\mathrm{~kg}]$ and $8.598\left[\mathrm{~kg} \cdot \mathrm{m}^{2}\right]$, respectively. The lengths of $l_{1}, l_{2}, l_{3}$, and $l_{4}$ shown in Figure 3 were $0.3[\mathrm{~m}], 0.3[\mathrm{~m}], 0.305[\mathrm{~m}]$, and $0.415[\mathrm{~m}]$, respectively. The mass and inertia of the manipulator were as provided in [26]. In the simulation calculation, we chose the grid points of $\left(x_{b}, y_{b}\right)$, that is, $\Delta x$ and $\Delta y$ as $0.02[\mathrm{~m}]$, the search area of $\left[x_{b}^{\min }, x_{b}^{\max }\right] \times\left[y_{b}^{\min }, y_{b}^{\max }\right]$ was $[0.7 \mathrm{~m}, 1.1 \mathrm{~m}] \times[0.3 \mathrm{~m}, 0.9 \mathrm{~m}]$, and the objective function defined by (9) was calculated at each point. Figure 4 shows the contour plot of the objective function $E_{\text {con }}$. It shows that the position of the minimum $E_{\text {con }}$ is $\left(x_{b}, y_{b}\right)=(0.82 \mathrm{~m}, 0.48 \mathrm{~m})$ and the value of $E_{\text {con }}$ is $8.28 \times$ $10^{5}\left[\mathrm{~N}^{2} \mathrm{~m}^{2} \mathrm{~s}\right]$. Figure 5 shows the motion process of the door in the planning time.

\section{Experimental Results}

5.1. Robot System. The mobile robot system (see Figure 6) consists of a 6-DOF modular manipulator produced by the SCHUNK Company, a four-wheeled platform with two drive wheels, and a two free wheels and two-finger gripper mounted on the arm wrist module. The 6-DOF modular manipulator comprises three types of modules, and each joint module consists of a brushless DC motor, a harmonic drive, a braking system, and an encoder. The mobile robot system is equipped with various types of sensors, including two six-axis force sensors and a camera. The two six-axis force sensors are mounted on the manipulator wrist module and the base module, respectively, to measure the mechanics date of the dooropening procedure. A joystick with associated force feedback control from the base-mounted 6-axis force/torque sensor is used for teleoperation. A camera is mounted on top of the frame to support 3D displays for continuous teleportation and object recognition. An API T3 (Automated Precision Inc.) laser tracker system was used to measure the base position in the experiment.

5.2. Controller Description. The structure of the proposed controller scheme is illustrated in Figure 7. The control 


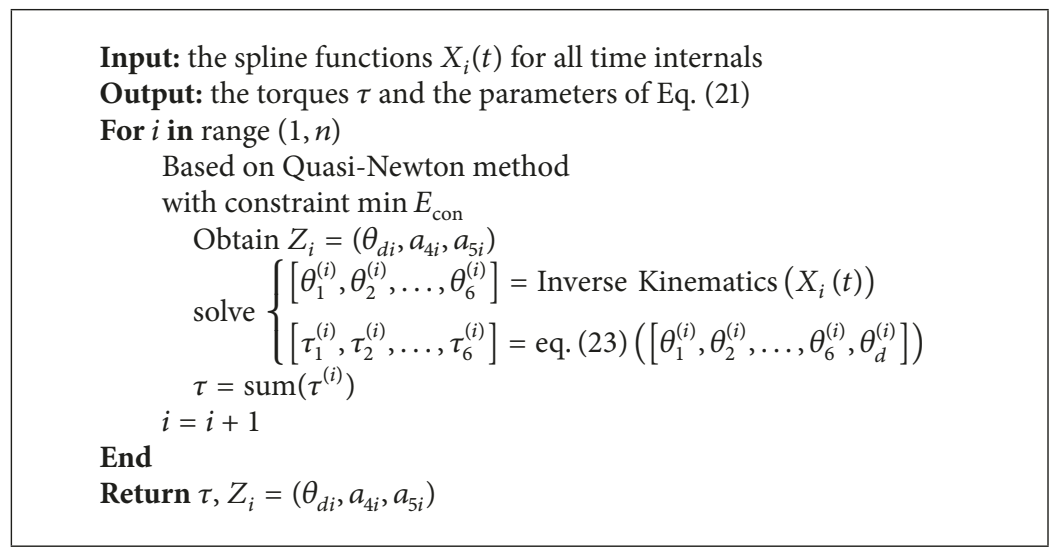

Algorithm 1: Select the optimal motion of door.

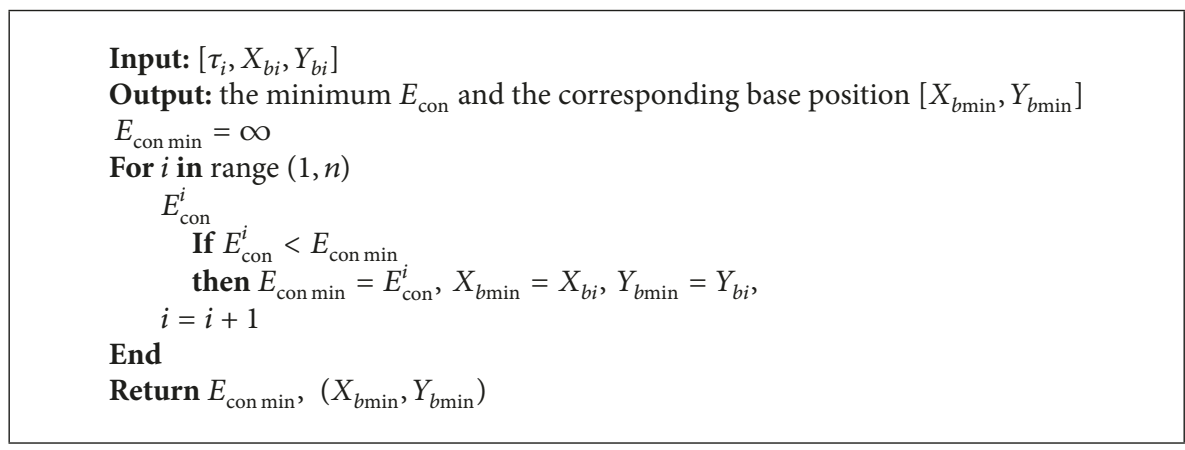

Algorithm 2: Search the optimal position of robot base.

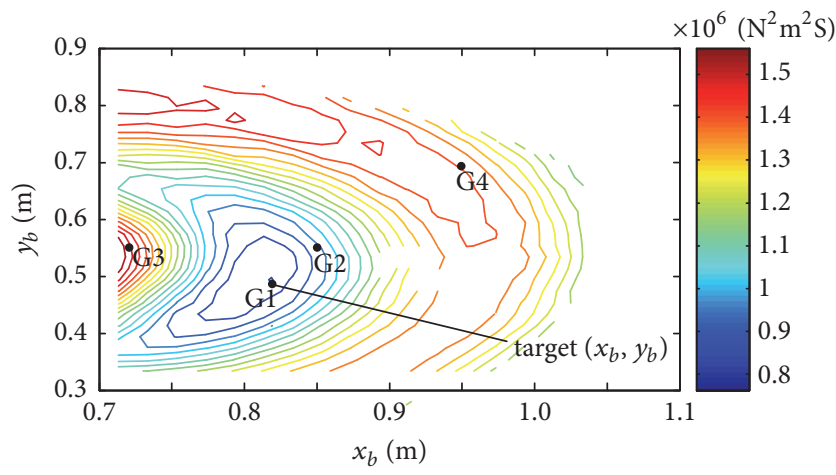

FIGURE 4: Objective for base position $\left(x_{b}, y_{b}\right)$.

system is shown in terms of the composition of the PDcomputed torque controller. The computed torque controller (CTC) uses a feedback linearization method. It is assumed that the desired motion trajectory for the manipulator is determined by a path planner [27]. The tracking error is defined as

$$
e(t)=\theta_{r}(t)-\theta(t)
$$

where $e(t)$ is the error of the plant, $\theta_{r}(t)$ is the desired input variable, which is the desired joint angular displacement in

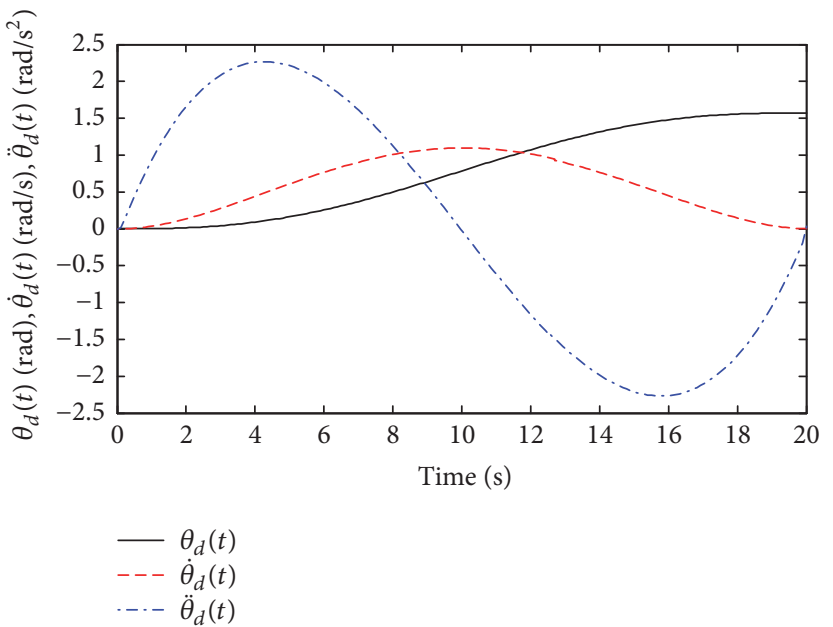

FIgURE 5: Optimal moment of the door.

the control system, and $\theta(t)$ is the actual joint angular displacement.

The control torque is described as

$$
\widetilde{\boldsymbol{\tau}}=\mathbf{V}(\theta, \dot{\theta}) \dot{\theta}+\mathbf{G}(\theta)+\mathbf{M}(\theta) \mathbf{u}
$$

The dynamic model in (19) is equivalent to a decoupled linear time-invariant system: 


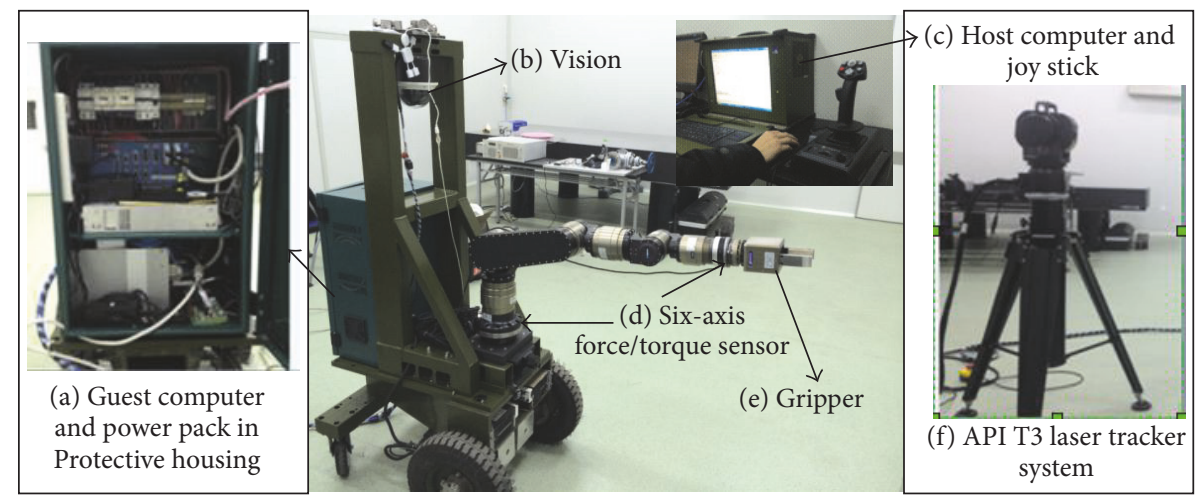

FIGURE 6: Software and hardware of the mobile manipulator.

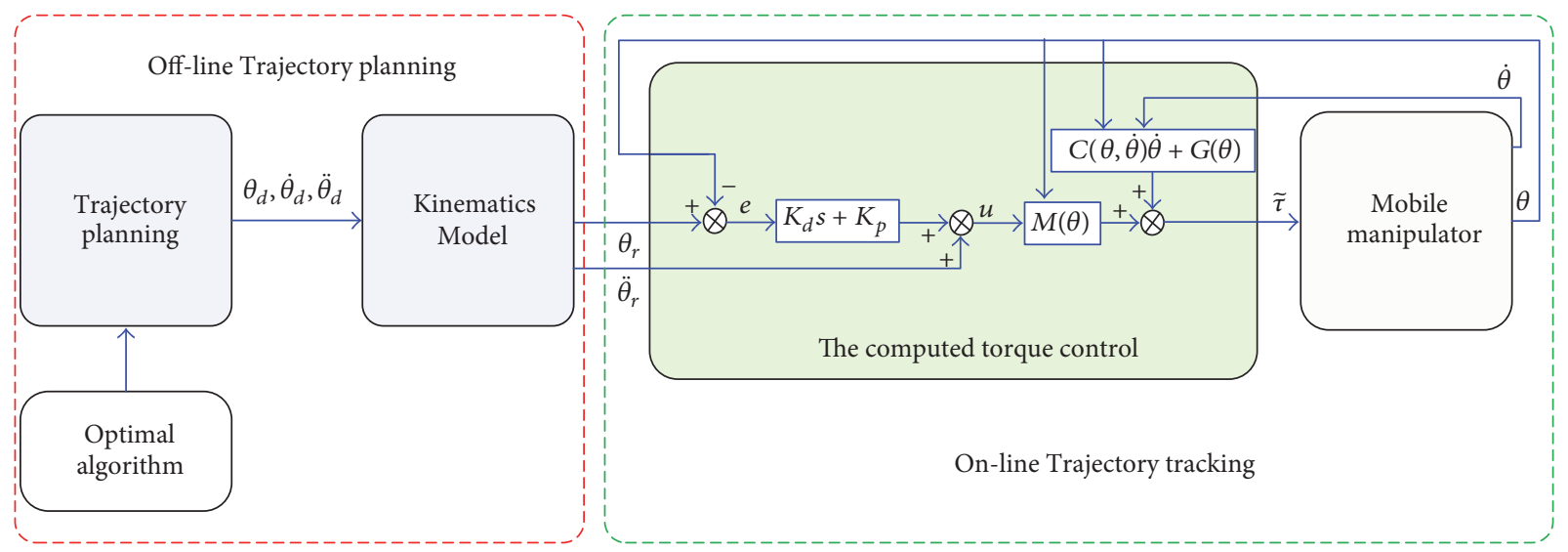

FIGURE 7: Block diagram of the computed torque controller.

$$
\ddot{\theta}=\mathbf{u} \text {. }
$$

Considering that the desired trajectory $\theta_{r}(t)$ is determined, $\dot{\theta}_{r}$ and $\ddot{\theta}_{r}$ are known. This is a nonlinear feedback control law that guarantees tracking of the desired dooropening trajectory. Selecting PD feedback for $u(t)$ results in the $\mathrm{PD}$-computed torque controller.

$$
\begin{aligned}
\mathbf{u} & =\ddot{\boldsymbol{\theta}}_{\mathbf{r}}+\mathbf{K}_{\mathbf{d}}\left(\dot{\boldsymbol{\theta}}_{\mathbf{r}}-\dot{\boldsymbol{\theta}}\right)+\mathbf{K}_{\mathbf{p}}\left(\boldsymbol{\theta}_{\mathbf{d}}-\boldsymbol{\theta}\right) \\
& =\ddot{\boldsymbol{\theta}}_{\mathbf{r}}+\mathbf{K}_{\mathbf{d}} \dot{\mathbf{e}}+\mathbf{K}_{\mathbf{p}} \mathbf{e}
\end{aligned}
$$

where $\mathbf{K}_{\mathbf{d}}$ and $\mathbf{K}_{\mathbf{p}}$ are the controller gains and are positive definite diagonal matrices, $\mathbf{K}_{\mathbf{p}}=\operatorname{diag}(8.2,10.4,8.5,7.4,12.5,9.0)$, $\mathbf{K}_{\mathbf{d}}=\operatorname{diag}(7.0,9.5,8.0,7.0,11.5,8.5)$. The closed-loop system equation is

$$
\ddot{\boldsymbol{\theta}}_{\mathbf{r}}+\mathbf{K}_{\mathbf{d}} \dot{\mathbf{e}}+\mathbf{K}_{\mathbf{p}} \mathbf{e}=0 .
$$

By substituting (27) into (25), we obtain the complete expression of the control law:

$$
\tilde{\boldsymbol{\tau}}=\mathbf{M}(\theta)\left(\ddot{\theta}_{r}+\mathbf{K}_{\mathbf{d}} \dot{e}+\mathbf{K}_{\mathbf{p}} e\right)+\mathbf{V}(\theta, \dot{\theta}) \dot{\theta}+\mathbf{G}(\theta) .
$$

5.3. Experimental Setup. The experiments were conducted in a simulated NPPs internal environment and a real NPPs fire door with a door closer was used. The trajectory during door-opening was calculated using the method described in Section 2.2. The entire door-opening procedure was realized using $\mathrm{C}++$ programming with a cycle of $20 \mathrm{~ms}$. Four groups of experiments using different base positions were conducted. In the first group, the base position, denoted by G1, was $\left(x_{b}=0.82 \mathrm{~m}, y_{b}=0.48 \mathrm{~m}\right)$. This is the point of the minimum objective function according to the numerical simulations. In the second group, denoted by G2, was $\left(x_{b}=0.85 \mathrm{~m}\right.$, $\left.y_{b}=0.55 \mathrm{~m}\right)$. In the third group, the base position, denoted by $\mathrm{G} 3$, was $\left(x_{b}=0.72 \mathrm{~m}, y_{b}=0.55 \mathrm{~m}\right)$. In the fourth group, the base position, denoted by G4, was $\left(x_{b}=0.95 \mathrm{~m}, y_{b}=0.7 \mathrm{~m}\right)$. The points G1, G2, G3, and G4 have been pointed and marked in Figure 4. G1 is the point of the minimum objective function as shown in Figure 4.

Figure 8 shows sequential pictures of the door-opening experiments. The duration of the door-opening procedure is $20 \mathrm{~s}$, which is the same as that the numerical simulation described in Section 4.3. The mobile robot system can measure the instantaneous current in the door-opening procedure. The torque of each joint was calculated using the values of the instantaneous current and (8). Therefore, the value of $E_{\text {con }}$ could be calculated in the various groups of experiments. The test data were acquired at a sampling frequency of $50 \mathrm{~Hz}$. The experiments were conducted more than three times under the same conditions to ensure the repeatability 


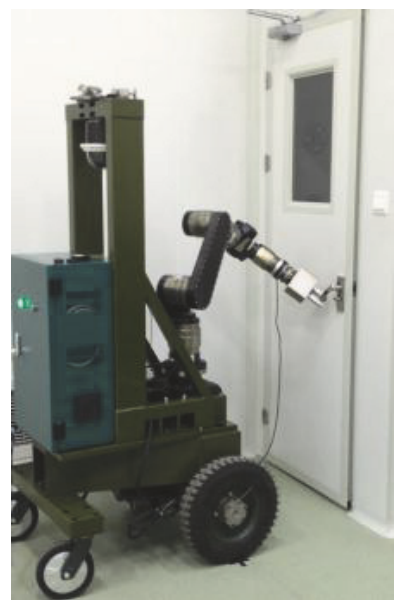

(a)

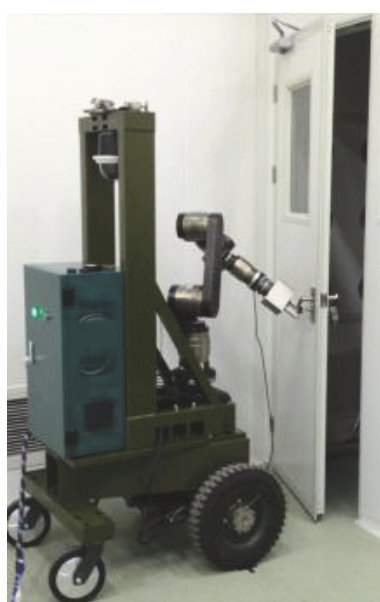

(b)

FIGURE 8: Door-opening experiment: (a) initial moments; (b) pulling the door.

and effectiveness of the experimental results. During the comparison of the energy consumption in the different groups of experiments, the end-effector grasped the door handle in the same positions according to the door-opening path planning provided in Section 2.

The performance parameters of the motors and actuating devices of joints are shown in Table 1 .

5.4. Comparison of the Different Base Positions. The matrix $\tau_{j}$ when the robot base is positioned at G1, G2, G3, and G4 can be calculated by using (8) and the performance parameters of the joints, shown in Table 1 . The values of $\sum_{j=1}^{6} \tau_{j}^{2}$ during the door-opening procedure are shown in Figure 9. The sums of the objective function for the different base positions are shown in Table 2.

The $E_{\text {con }}$ curves shown in Figure 9 demonstrate that energy consumption is optimized at base position G1. Figure 9 shows that the torque is increased during the start and end of the door-opening procedure because of the resistance of the door handle and the door closer. It can be clearly seen in Figure 9 that, in comparison with other selected positions, over time G1 is better than the other selected base positions minimizing the objective function, not only in terms of the accumulated value of $E_{\text {con }}$, shown in Table 2, but also in terms of sustaining the lowest value of $\sum_{j=1}^{6} \tau_{j}^{2}$. This verifies the effectiveness of the proposed algorithm. The experiment value of $E_{\text {con }}$ is larger than the simulation value at the same base position (G1), because the simulation only considered the mechanical power of (6); however, the experiment value included the generated mechanical power, heat power, and power losses due to factors such as friction.

Figures 10 and 11 show the optimal trajectories of the joint angle $\theta_{i}$ and the torque $\tau$ of each joint for G1. It can be seen in Figure 11 that the values of the joints' torques $\tau_{2}, \tau_{3}$ are larger than those of the other torques. Figure 10 shows that in the motion during the door-opening procedure the angle degree of Joints 2 and 3 is larger than that of the other joints.

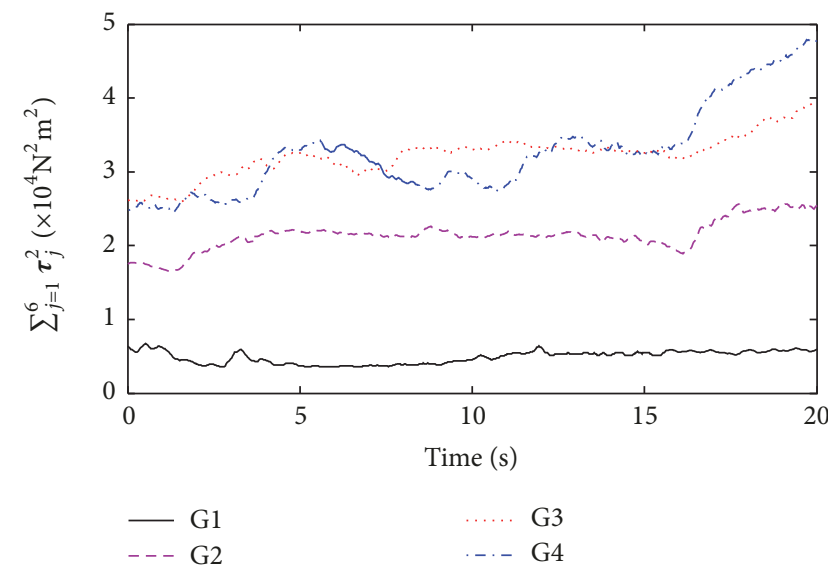

FIGURE 9: Comparison of the square sums of joint torque at the different base positions.

\section{Conclusion}

In this paper, we proposed a novel energy consumption optimization scheme for a mobile manipulator executing the door-opening task. Focusing on the power consumption of the manipulator during the entire task period, we chose the quadric terms of the joint torques as the objective function. Furthermore, in this study a two-step optimization procedure was developed to solve the corresponding joint trajectories of the manipulator. In the first step, the feasible base positions of the manipulator are decentralized into a grid in order to simplify the entire optimization process. A piecewise fifthorder polynomials over time is utilized to parameterize the rotational motion of the door. By applying the Quasi-Newton method, the local optimal trajectories of the manipulator are obtained for a given base position. In the second step, the optimal base position is attained via searching the decentralized grid of the feasible base positions. The numerical results when the proposed method was applied showed that 
TABLE 1: Performance parameters of drive devices and actuating devices of joints.

\begin{tabular}{lccccc}
\hline Joint of arm $j$ & $U_{\text {in }} /(\mathrm{V})$ & $K_{m j} /(\mathrm{mN} \cdot \mathrm{m})$ & $i_{j}$ & $R_{j} /(\Omega)$ & 1.5 \\
\hline Joints 1 and 2 & 24 & 31.4 & 596 & 0.6 & 0.6 \\
Joints 3 and 4 & 24 & 38 & 625 & 0.85 \\
Joints 5 and 6 & 24 & 16 & 552 & 0.1 \\
\hline
\end{tabular}

TABLE 2: Value of $E_{\text {con }}$ at different base positions.

\begin{tabular}{lcccc}
\hline Objective function & G1 & G2 & G3 & G4 \\
\hline$E_{\text {con }}\left(\mathrm{N}^{2} \mathrm{~m}^{2} \mathrm{~s}\right)$ & $2.97 \times 10^{6}$ & $1.65 \times 10^{7}$ & $2.79 \times 10^{7}$ & $2.95 \times 10^{7}$ \\
\hline
\end{tabular}

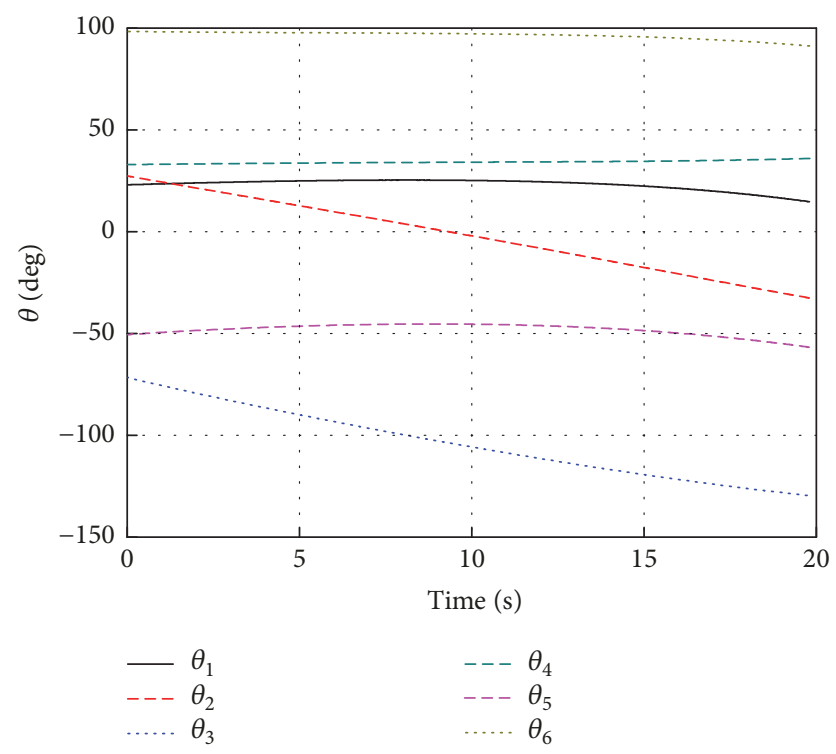

Figure 10: Angle of each joint (G1).

energy consumption was optimized at base position G1. The experimental results for door-opening at the different base positions demonstrate the effectiveness of the method proposed in this study. The proposed method will be useful for the development of the NPP rescue robots.

\section{Conflicts of Interest}

The authors declare no potential conflicts of interest with respect to the research, authorship, and/or publication of this article.

\section{Acknowledgments}

This work was supported by the National Key Basic Research Development Plan Project of China (973) (2013CB035502), Project supported by the Foundation for Innovative Research Groups of the National Natural Science Foundation of China (Grant no. 51521003), Research Project of State Key Laboratory of Mechanical System and Vibration (MSV201610), Harbin Talent Program for Distinguished Young Scholars

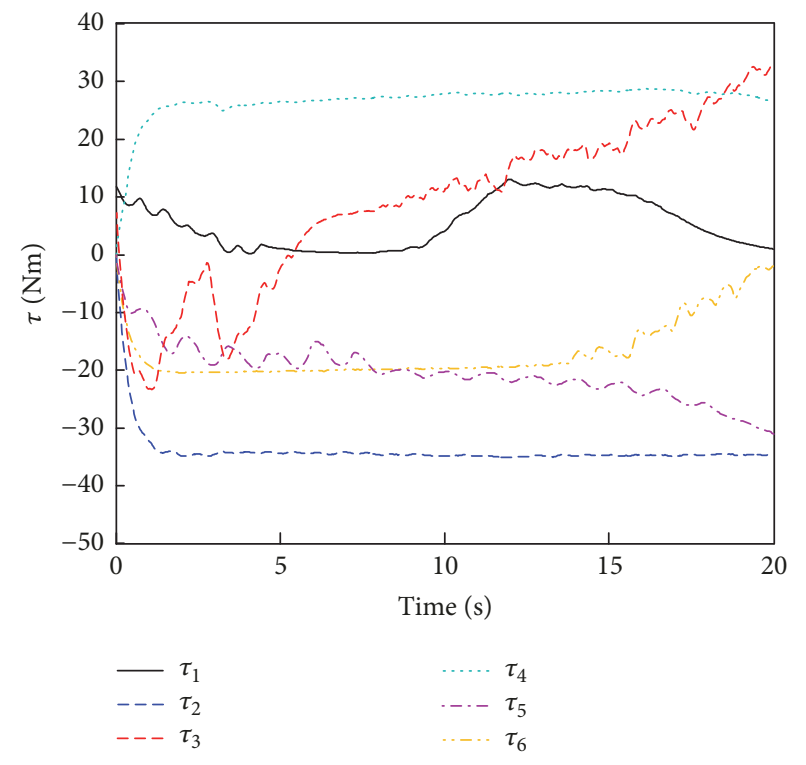

FIgURE 11: Torque of each joint (G1).

(no. 2014RFYXJ001), Heilongjiang Province Higher Education Project of Basic Scientific Research (2017-KYYWF0568), Harbin Applied Technology Project of Research and Development (2015RQQXJ081), and “111” Project (B07018).

\section{References}

[1] E. Sciubba, "Fukushima: There are lessons to be learnt, on both sides," Energies, vol. 4, no. 5, pp. 818-825, 2011.

[2] N. Sasaki, T. Owari, and F. E. Putz, "Time to substitute wood bioenergy for nuclear power in Japan," Energies, vol. 4, no. 7, pp. 1051-1057, 2011.

[3] J. Ma, J. Luo, H. Pu, Y. Peng, S. Xie, and J. Gu, "Design, simulation and manufacturing of a tracked robot for nuclear accidents," in Proceedings of the 2014 IEEE International Conference on Robotics and Biomimetics, IEEE ROBIO 2014, pp. 1828-1833, Indonesia, December 2014.

[4] G. Wu, P. Ju, X. Song, C. Xie, and W. Zhong, "Interaction and coordination among nuclear power plants, power grids and their protection systems," Energies, vol. 9, no. 4, article no. 306, 2016.

[5] M. DeDonato, V. Dimitrov, R. Du et al., "Human-in-the-loop control of a humanoid robot for disaster response: A report from the DARPA robotics challenge trials," Journal of Field Robotics, vol. 32, no. 2, pp. 275-292, 2015.

[6] H. A. Yanco, A. Norton, W. Ober, D. Shane, A. Skinner, and J. Vice, "Analysis of human-robot interaction at the DARPA robotics challenge trials," Journal of Field Robotics, vol. 32, no. 3, pp. 420-444, 2015. 
[7] K. Nagatani and S. Yuta, "Designing a behavior to open a door and to pass through a door-way using a mobile robot equipped with a manipulator," in Proceedings of the IEEE/RSJ/GI International Conference on Intelligent Robots and Systems. Part 3 (of 3), pp. 847-853, September 1994.

[8] K. Nagatani and S. Yuta, "Designing strategy and implementation of mobile manipulator control system for opening door," in Proceedings of the 1996 13th IEEE International Conference on Robotics and Automation. Part 1 (of 4), pp. 2828-2834, April 1996.

[9] K. Nagatani and S. Yuta, "Experiment on opening-door-behavior by an autonomous mobile robot with a manipulator," in Proceedings of the 1995 IEEE/RSJ International Conference on Intelligent Robots and Systems. Part 3 (of 3), pp. 45-50, August 1995.

[10] L. Peterson, D. Austin, and D. Kragic, "High-level control of a mobile manipulator for door opening," in Proceedings of the Proceedings. 2000 IEEE/RSJ International Conference on Intelligent Robots and Systems (IROS 2000), pp. 2333-2338, Takamatsu, Japan.

[11] W. Chung, C. Rhee, Y. Shim, H. Lee, and S. Park, "Door-Opening control of a service robot using the multifingered robot hand," IEEE Transactions on Industrial Electronics, vol. 56, no. 10, pp. 3975-3984, 2009.

[12] S. Ahmad and G. Liu, "A door opening method by modular reconfigurable robot with joints working on passive and active modes," in Proceedings of the 2010 IEEE International Conference on Robotics and Automation, ICRA 2010, pp. 1480-1485, USA, May 2010.

[13] S. Ahmad, H. Zhang, and G. Liu, "Multiple working mode control of door-opening with a mobile modular and reconfigurable robot," IEEE/ASME Transactions on Mechatronics, vol. 18, no. 3, pp. 833-844, 2013.

[14] H. Zhang, Y. Liu, and G. Liu, "Multiple mode control of a compact wrist with application to door opening," Mechatronics, vol. 23, no. 1, pp. 10-20, 2013.

[15] S. Kobayashi, Y. Kobayashi, Y. Yamamoto et al., "Development of a door opening system on rescue robot for search "UMRS2007", in Proceedings of the SICE Annual Conference 2008 - International Conference on Instrumentation, Control and Information Technology, pp. 2062-2065, Japan, August 2008.

[16] E. Klingbeil, A. Saxena, and A. Y. Ng, "Learning to open new doors," in Proceedings of the 23rd IEEE/RSJ 2010 International Conference on Intelligent Robots and Systems, IROS 2010, pp. 2751-2757, Taiwan, October 2010.

[17] Y. Karayiannidis, C. Smith, F. E. Viña, P. Ögren, and D. Kragic, “"Open sesame!" adaptive force/velocity control for opening unknown doors," in Proceedings of the 25th IEEE/RSJ International Conference on Robotics and Intelligent Systems, IROS 2012, pp. 4040-4047, Portugal, October 2012.

[18] F. Endres, J. Trinkle, and W. Burgard, "Learning the dynamics of doors for robotic manipulation," in Proceedings of the 2013 26th IEEE/RSJ International Conference on Intelligent Robots and Systems: New Horizon, IROS 2013, pp. 3543-3549, Japan, November 2013.

[19] J. Yuan, G. Liu, and B. Wu, "Power efficiency estimation-based health monitoring and fault detection of modular and reconfigurable robot," IEEE Transactions on Industrial Electronics, vol. 58, no. 10, pp. 4880-4887, 2011.

[20] L.-P. Luo, C. Yuan, R.-J. Yan et al., “Trajectory planning for energy minimization of industry robotic manipulators using the Lagrange interpolation method," International Journal of
Precision Engineering and Manufacturing, vol. 16, no. 5, pp. 911917, 2015 .

[21] G. Field and Y. Stepanenko, "Iterative dynamic programming: an approach to minimum energy trajectory planning for robotic manipulators," in Proceedings of the IEEE International Conference on Robotics and Automation, vol. 3, pp. 2755-2760, IEEE, Minneapolis, Minn, USA, April 1996.

[22] Y. Liu, L. Liang, H. Han, and S. Zhang, "A method of energyoptimal trajectory planning for palletizing robot," Mathematical Problems in Engineering, vol. 2017, 10 pages, 2017.

[23] J. Li, J. Tao, L. Ding, H. Gao, Z. Deng, and K. Xia, “Twisting door handles and pulling open doors with a mobile manipulator," in Proceedings of the IEEE International Conference on Robotics and Biomimetics, IEEE-ROBIO 2015, pp. 686-691, China, December 2015.

[24] H. Zhuang, H. Gao, L. Ding, Z. Liu, and Z. Deng, "Method for analyzing articulated torques of heavy-duty six-legged robot," Chinese Journal of Mechanical Engineering, vol. 26, no. 4, pp. 801-812, 2013.

[25] A. Vergnano, C. Thorstensson, B. Lennartson et al., "Modeling and optimization of energy consumption in cooperative multirobot systems," IEEE Transactions on Automation Science and Engineering, vol. 9, no. 2, pp. 423-428, 2012.

[26] L. Ding, K. Xia, H. Gao, G. Liu, and Z. Deng, "Robust adaptive control of door opening by a mobile rescue manipulator based on unknown-force-related constraints estimation," Robotica, vol. 36, no. 1, pp. 119-140, 2018.

[27] S. Soltani and F. Piltan, "Design artificial nonlinear controller based on computed torque like controller with tunable gain," World Applied Sciences Journal, vol. 14, no. 9, pp. 1306-1312, 2011. 


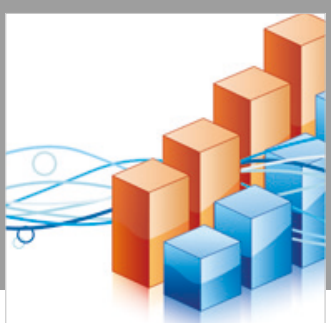

Advances in

Operations Research

\section{-n-m}
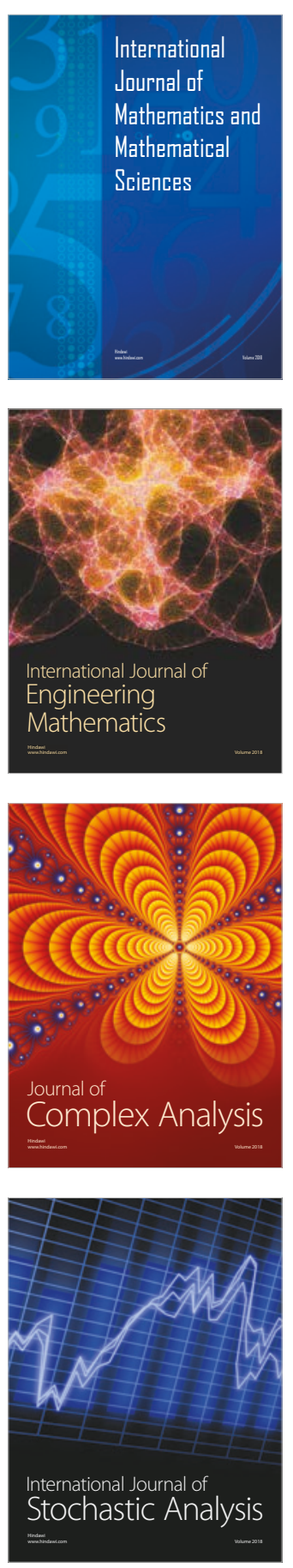
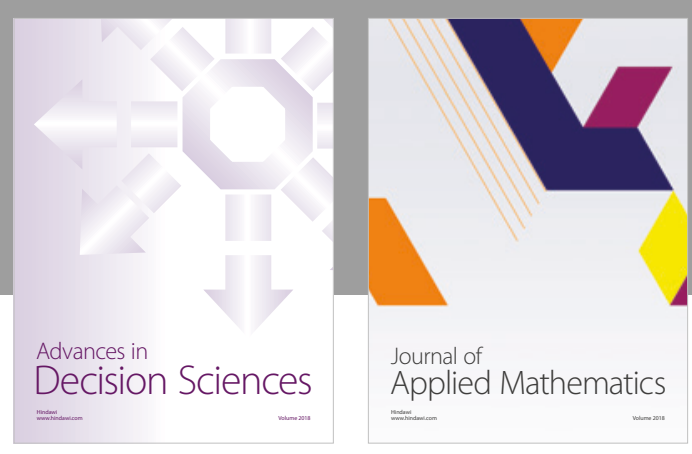

Journal of

Applied Mathematics
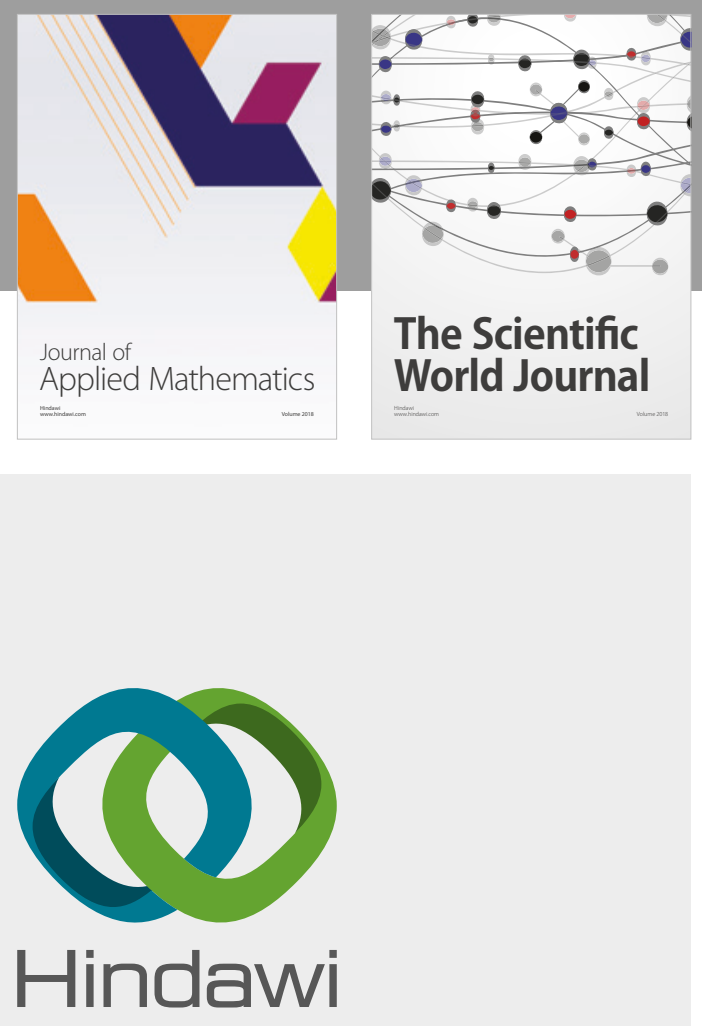

Submit your manuscripts at

www.hindawi.com

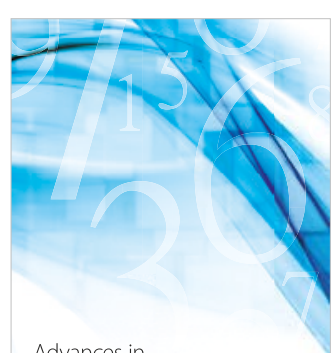

Advances in
Numerical Analysis
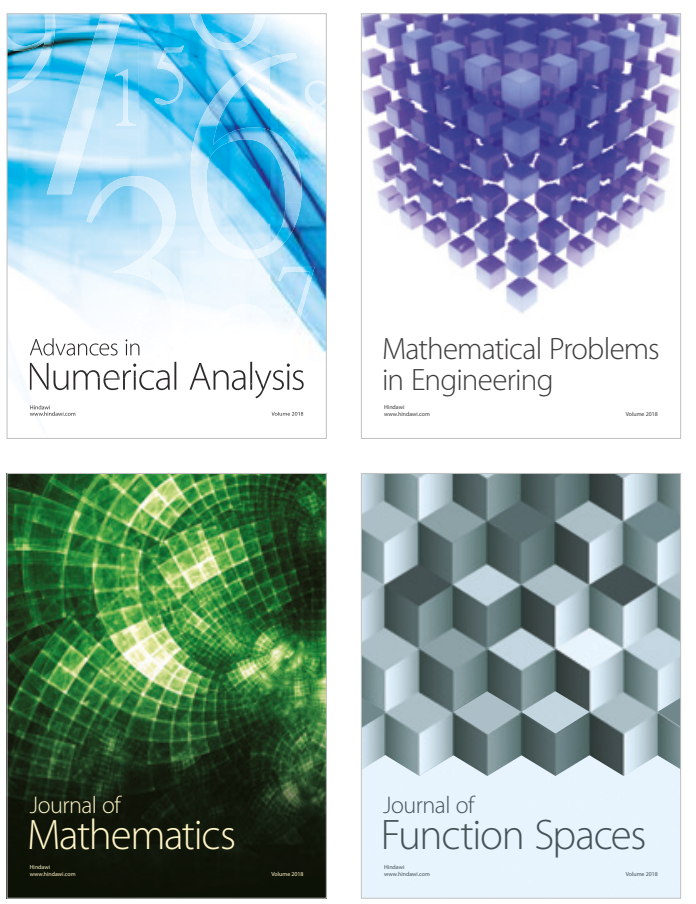

Mathematical Problems in Engineering

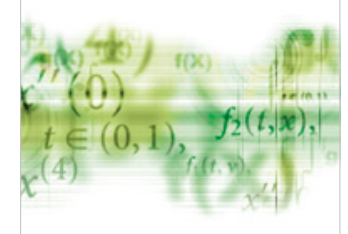

International Journal of

Differential Equations

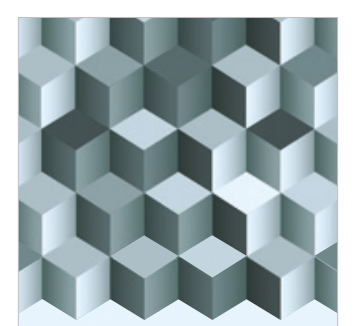

Journal of

Function Spaces

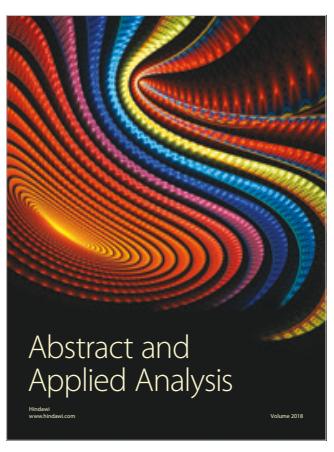

The Scientific

World Journal

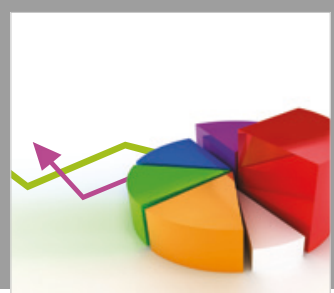

Journal of

Probability and Statistics
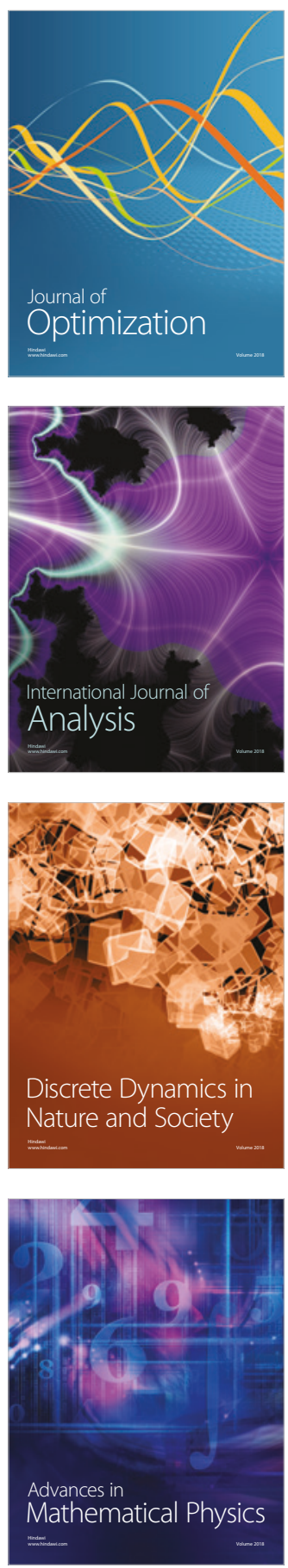